\title{
Response to a Dolutegravir-based Regimen in an HIV- Infected Woman with Multiple Comorbidities and a Highly Resistant Strain
}

\author{
Amedeo Capetti ${ }^{1{ }^{*}}$, Laura Carenzi ${ }^{1}$, Noemi Astuti ${ }^{1}$, Valeria Cozzi ${ }^{2}$, Valeria Micheli ${ }^{3}$, Maria Vittoria Cossu ${ }^{1}$ \\ ${ }^{1} 1^{\text {st }}$ Division of Infectious Diseases, Luigi Sacco University Hospital, Milano, Italy \\ ${ }^{2}$ Clinical Pharmacology Unit, Luigi Sacco University Hospital, Milano, Italy \\ ${ }^{3}$ Clinical Microbiology Unit, Luigi Sacco University Hospital, Milano, Italy \\ *Corresponding author: capame@hotmail.com
}

Received February 27, 2015; Revised May 01, 2015; Accepted June 10, 2015

\begin{abstract}
HIV-infected patients have a higher burden of comorbidities than the general population and drug-drug interactions limit the choice of antiretroviral compounds, also limiting the possibility of drug sequencing in case of toxicity or failure. In our patient, affected by HIV-related pulmonary hypertension (HIV-PAH), the use of sildenafil and ambrisentan excluded the possibility of using two of the main classes of antiretrovirals, protease inhibitors (PIs) and non-nucleoside reverse transcriptase inhibitors (NNRTIs). Having failed a combination regimen based on raltegravir, while awaiting the results of genotypic testing for drug resistance she was switched to dolutegravir, a brand new integrase strand transfer inhibitor (INSTI) with high genetic barrier, and in one month her HIV-1 viremia dramatically dropped to undetectable levels. The genotypic test revealed resistance to all the drugs she was taking including dolutegravir. The backbone was changed to a dual regimen including rilpivirine and viral suppression was maintained at three months. Unprecedented pharmacokinetic data are provided for the two antiviral drugs in combination with sildenafil and ambrisentan.
\end{abstract}

Keywords: HIV, PAH, dolutegravir, sildenafil, ambrisentan, resistance

Cite This Article: Amedeo Capetti, Laura Carenzi, Noemi Astuti, Valeria Cozzi, Valeria Micheli, and Maria Vittoria Cossu, "Response to a Dolutegravir-based Regimen in an HIV-Infected Woman with Multiple Comorbidities and a Highly Resistant Strain.” American Journal of Medical Case Reports, vol. 3, no. 7 (2015): 201-204. doi: 10.12691/ajmcr-3-7-5.

\section{Introduction}

Pulmonary arterial hypertension (PAH) is a rare disease, that left untreated has an estimated median survival of 2.8 years [1]. Survival, however, has dramatically improved in the last decade due to advances in treatment options [2]. Among HIV-infected subjects with $\mathrm{PAH}$, survival has improved from $58 \%, 32 \%$, and $21 \%$ at 1,2 , and 3 years, respectively, in the 1990 s, to $88 \%, 72 \%$, and $63 \%$ at 1,3 , and 5 years, respectively [3]. Antiretroviral therapy improves survival, but specific PAH treatment limits the choice of antiretrovirals. Moreover, antihypertensive drugs may cause liver damage or circulatory effects that can sum to antiretroviral therapy side effects and toxicity. Also, in several subjects, the presence of HCV coinfection or liver decompensation adds the burden of portopulmonary shunts with porto-pulmonary arterial hypertension. Therefore an HIV-infected subject is a particularly delicate person, in whom any error may lead to serious consequences.

\section{Case Report}

A 47 year old hispanic female was diagnosed and treated for Hodgkin lymphoma in 2000, underwent total hysterectomy for uterine cancer in 2006, and in September 2010 had an episode of pneumonia, after which pulmonary arterial hypertension (PAH) was diagnosed. Looking for a possible aetiology, an HIV antibody test was performed and resulted positive in December, 2010. The patient had never tested for HIV before and had had heterosexual contacts. Her plasma HIV-1 RNA was 34.030 copies $/ \mathrm{mL}$ and she had 174 CD4+ T lymphocytes/mmc. Her mean pulmonary arterial pressure (PAPs) was $100 \mathrm{mmHg}$ at cardiac ultrasound, right heart catheterization showed severe pulmonary hypertension (84 mmHg) with increased vascular and total - systemic and pulmonary - resistance, with a slightly reduced cardiac output (3.25 L/min). CT scan, x-rays, scintigraphy and blood work did not reveal other causes. A thyroid suspect nodule was secondarily found during the assessments for PAH. Referred to our hospital, combination antiretroviral therapy (cART) with tenofovir/emtricitabine plus raltegravir was started on December 13, 2010, together with anti-hypertensive treatment with sildenafil $20 \mathrm{mg}$ q8h, while the thyroid nodule was aspirated, yielding the diagnosis of papillary thyroid carcinoma, that was surgically removed. 
Radiotherapy was performed and subsequently the patient started levothyroxine $100 \mathrm{mg} /$ day. One year later a cardiac ultrasound revealed a hypokinetic right ventricle with further increase of pulmonary pressure $(112 \mathrm{mmHg})$ and initial rectilinearization of the interventricular septum. Ambrisentan $5 \mathrm{mg}$ /day was added with important reduction of hypertension (PAPs 45 mmHg, July, 2012). The patient was classified as NYHA III. The situation stabilized in the following years, but in summer 2014 her adherence to therapy worsened and she did not present for her scheduled blood work in July. In October, 2014, a stress test revealed serious functional aerobic deficit with an important deficiency of the pulmonary perfusion, and the 6 minutes' walking test had worsened (400 meters). The blood work performed the $19^{\text {th }}$ of November, 2014 showed immunovirologic failure, with HIV RNA rising to 4661 copies/mL and CD4+ T cells falling to 292/mmc. A genotypic test was requested and in the meanwhile, having very little choice available, only raltegravir was changed to dolutegravir $50 \mathrm{mg} \mathrm{q} 12 \mathrm{~h}$, waiting for the results to make a rational treatment change. At the visit of January, 7, 2015, the genotypic test revealed the presence of K65R and $\mathrm{M} 184 \mathrm{~V}$ resistance-associated mutations (RAMs) in rev causing high-level resistance to all nucleoside/nucleotide analogues except zidovudine while the sequencing of the integrase region yielded RAMs L74M, G140S and Q148H, conferring high-level resistance to all INSTIs; both the TRUGENE Guidelines ${ }^{\mathrm{TM}}$ Rules 17.0 and the Stanford HIVdb algorithms were concordant in data interpretation (Figure 1). Despite this apparent complete resistance to the modified regimen - as well as to the previous one, the HIV-1 RNA plasma level had decreased in four weeks to completely undetectable, therefore it was decided to maintain dolutegravir, switching from tenofovir/emtricitabine to rilpivirine, the only NNRTI free from drug-drug interactions with her comedications. At week 12, the patient maintains optimal viral suppression, her immunity is recovering ( $+24 \mathrm{CD} 4+\mathrm{T}$ cells/mmc, $+2,5 \%$, Figure 2 ) and the subject tolerates the new regimen very well. Plasma minimum concentrations $\left(\mathrm{C}_{\min }\right)$ of dolutegravir (12h) was quite high, while that of rilpivirine (24h) was perfectly in the range. No ECG alterations were recorded, as dolutegravir does not impact on QT prolongation, however some concern may be raised (Figure 3). No cancer recurrence has developed up to date. The 6 minutes' walking test has improved again (from 400 to 435 meters).
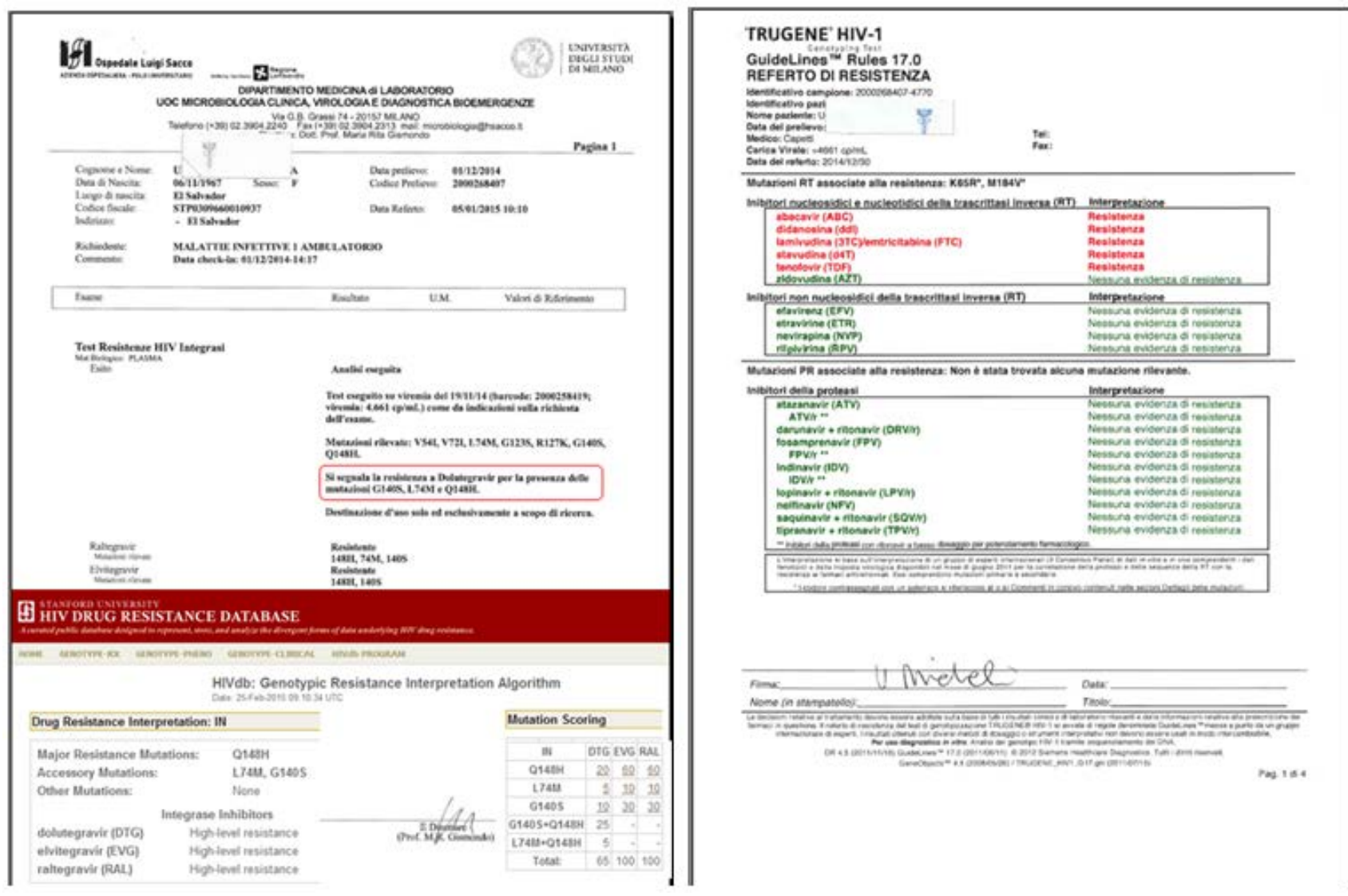

Figure 1. Genotypic testing showed complete resistance to nucleoside analogues and INSTIs

\section{Discussion}

The pathogenesis of HIV-related PAH is complex and not entirely understood. Many factors, as viral proteins, HIV-related inflammation, coinfections with other microrganisms, and genetic susceptibility were considered to contribute to HIV-related PAH. Recently, a direct role of HIV infection to endothelial damage through the release of different inflammatory mediators such as endothelin-1 has been suggested [4]. Even the effect of antiretroviral therapy on pulmonary artery pressure has been an area of controversy. A recent study suggested the possible role of antiretroviral therapy in improving pulmonary artery pressure in HIV/AIDS patients if started at early stages (WHO classes I and II). Any significant effect was observed at more advanced stages [5]. In our 
case report, the patient showed a worsening in pulmonary perfusion and in the 6 minutes' walking test at the moment of viral failure and an improvement after viral suppression with the new INSTI, suggesting a possible direct virologic role in $\mathrm{PAH}$ evolution. However, since viral failure was a consequence of reduced compliance to HIV medications, a poor compliance to specific drugs may provide a simpler explanation.

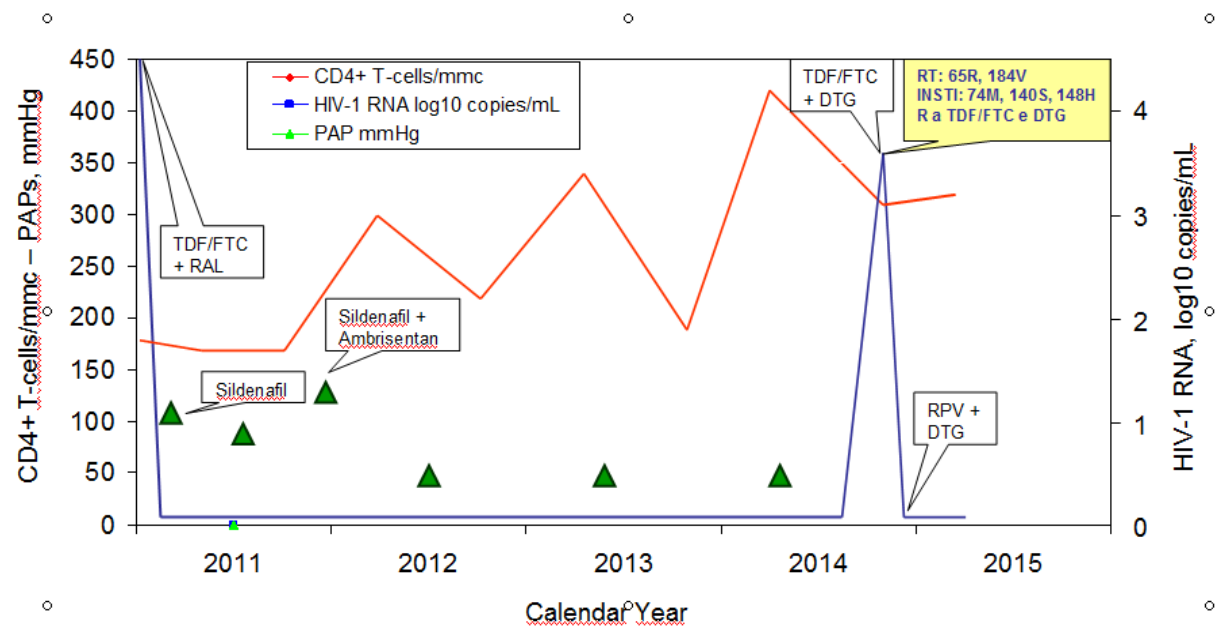

Figure 2. Evolution of HIV-1 RNA, CD4 cell counts, resistance mutations and pulmonary arterial pressure

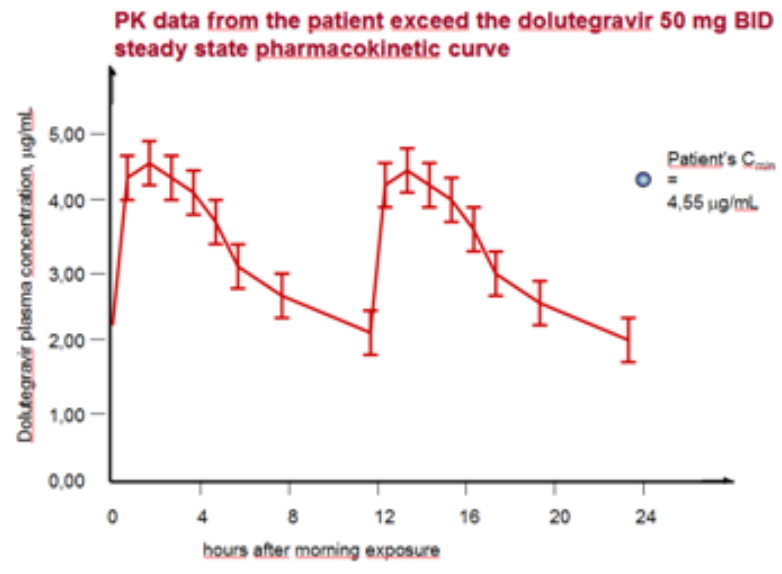

AUC $75,1 \mu \mathrm{g}, \mathrm{h} / \mathrm{mL}: \mathrm{C}_{\max } 4,15 \mu \mathrm{g} / \mathrm{mL}, \mathrm{C}_{\min } 2,12 \mu \mathrm{g} / \mathrm{mL}$

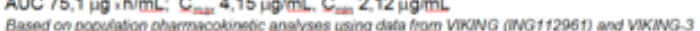

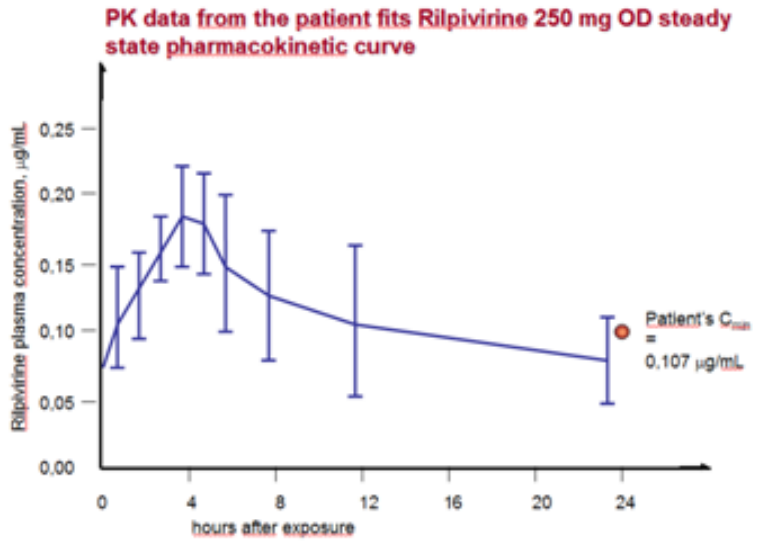

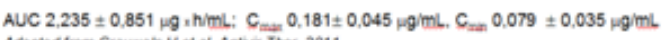
Adanted toon Craviels $\mathrm{H}$ ef al, Antivi Ther, 2011

Figure 3. Dolutegravir and rilpivirine Cmin as compared with the steady-state population PK curves

Vasodilator and anti-endothelin receptor A drugs are essential in the treatment of $\mathrm{PAH}$, but can limit the choice of antiretroviral drugs, due to possible drug-drug interactions. Sildenafil is a phosphodiesterase 5 inhibitor (PDE5 inhibitor) commonly used in PAH at high doses. At these doses is contraindicated with antiretroviral regimens based on protease inhibitors (PI) or non nucleoside inverse trascriptase inhibitors (NNRTI). Coadministration with PIs is expected indeed to increase sildenafil blood concentrations, possibly resulting in adverse reactions [6]. On the other hand, coadministration with NNRTIs (except rilpivirine) may lead to a decrease in sildenafil blood concentrations, with possible reduction in clinical response. This is true especially for etravirine, that leads to a decrease in sildenafil AUC by $57 \%$ and in Cmax by $45 \%$ [7]. No data are available for efavirenz and nevirapine, but similar pharmacokinetic interactions are expected. Ambrisentan and bosentan are endothelin receptor A (ETa) antagonists used for PAH, metabolized by the hepatic cytochrome 3A (CYP3A). Thereby, the concomitant use of antiretrovirals inhibiting CYP3A, as for example PIs, or inducing CYP3A, as for example
NNRTIs, may lead to an increase or reduction in ETa antagonists blood concentration [8]. Moreover, antihypertensive drugs may cause liver toxicity or circulatory effects that can sum to antiretroviral therapy side effects [9].

Among antiretrovirals, the INSTI class presents a favorable metabolic and pharmacokinetic profile, except for elvitegravir that needs to be boosted by the CYP3A4 inhibitor cobicistat. Raltegravir and dolutegravir (DTG) may represent thereby a good choice in patients with comorbidities, to limit the potential risk of drug-drug interactions and the amount of toxicity.

In clinical trials on naive patients, $50 \%$ of subjects failing raltegravir developed raltegravir RAMs, and strains harboring more than two RAMs were rare [10]. The presence of single INSTI RAMs (Q148H/R/K, N155H, $\mathrm{Y} 143 \mathrm{R} / \mathrm{H} / \mathrm{C}$, E92Q e T66I) does not reduce the in vitro susceptibility to dolutegravir [11,12], however, when combined with at least two secondary mutations, a Q148 RAM leads to a 5-10 fold (or more) susceptibility reduction to DTG. The Viking study is a phase III randomized clinical trial, in which ARV experienced 
patients harboring raltegravir and/or elvitegravir resistance were randomized to DTG $50 \mathrm{mg}$ twice daily or placebo plus the failing regimen for 7 days (functional monotherapy phase) [13]. At day 8, all patients switched to open-label DTG $50 \mathrm{mg}$ BID plus optimized background therapy ( $\geq 1$ fully active drug). The study resulted in a day 8 plasma HIV-1-RNA mean change from baseline of $-1.43 \log 10 \mathrm{c} / \mathrm{mL}$ and in a $69 \%$ virologic suppression rate (VL $<50 \mathrm{c} / \mathrm{mL}$ ) at week 24. Baseline INI resistance (Q148 $+\geq 2$ associated mutations) and viral load were highly significant predictors for week 24 response. The odds of achieving HIV-1 RNA $<50 \mathrm{c} / \mathrm{mL}$ were $63 \%$ lower for every 2-fold increase in DTG fold change in 50\% inhibitory concentration (FC) and about $80 \%$ lower for every 10-fold increase in baseline HIV-1 RNA. The odds of achieving virologic undetectability resulted moreover $96 \%$ lower in subjects with virus harboring Q148 $+\geq 2$ mutations compared with those with no evidence of Q148 mutations.

Our patient's genotypic test showed mutations conferring resistance to almost all nucleoside analogues $(65 \mathrm{R}, 184 \mathrm{~V})$ and mutations in the integrase region $(148 \mathrm{H}$, 54I, 72I, 74M, 123S, $127 \mathrm{~K}, 140 \mathrm{~S})$ that led to a reduction in the sensitivity to DTG. Of note, only 8 patients in Viking study had total resistance to nucleoside analogues (backbone genotypic susceptibility score $=0$ ) and virologic success at 24 weeks was achieved in 4 of the 8 subjects. As seen in the first days of the Viking study, in our case report DTG has led after 1 month to optimal suppression of viral replication (with a 2,11 $\log 10$ copies/mL drop in viremia), despite the almost total inefficacy of the nucleoside backbone. The reduced impact of nucleoside backbone strongly supports the independent antiviral activity of DTG.

Once resistance to nucleoside analogues was acknowledged, our patient was switched to a dual treatment with dolutegravir and rilpivirine, allowing the maintenance of virologic suppression. This appears to be a simple regimen, very promising for the DTG high genetic barrier and for the pharmacokinetic profile of both drugs, that may deeply improve the patient's compliance and quality of life in the future [14]. Dolutegravir Cmin was more than twice the expected level, and this deserves a future double check, to understand whether a dose reduction should be performed. A limit in our case report is the lack of pharmacokinetic data for sildenafil and ambrisentan. However, no dose-dependent adverse events occurred, to suggest a significant change in blood concentration.

\section{Conclusion}

Patients affected with HIV-PAH have a limited treatment choice as compared with the general HIVinfected population and a higher mortality rate. Dolutegravir, due to its high genetic barrier and low potential for drug-drug interactions, is a very interesting option in this setting. Rilpivirine, too, was not affected by the use of specific PAH drugs. Possible future long-acting formulations of these drugs are therefore adequate to this subgroup of patients.

\section{References}

[1] D'Alonzo GE, Barst RJ, Ayres SM, Bergofsky EH, Brundage BH, Detre KM, Fishman AP, Goldring RM, Groves BM, Kernis JT, et al. Survival in patients with primary pulmonary hypertension. Results from a national prospective registry. Ann Intern Med. 1991; 115(5): 343-349.

[2] Baldi F, Fuso L, Arrighi E, and Valente S. Optimal management of pulmonary arterial hypertension: prognostic indicators to determine treatment course. Therapeutics and Clinical Risk Management 2014:10 825-839.

[3] Barnett CF and Hsue PY: Human Immunodeficiency VirusAssociated Pulmonary Arterial Hypertension. Clin Chest Med. 2013; 34(2): 283-292.

[4] Feijoo MQ et al. Relationship between endothelin-1 levels and pulmonary arterial hypertension in HIV-infected patients. AIDS. 2014 Nov 28; 28(18):2693-9.

[5] Pal J, et al. Effect of antiretroviral therapy on pulmonary hypertension in HIV patients J Indian Med Assoc. 2013 Dec; 111(12):845-6, 849.

[6] Muirhead GJ, Wulff MB, Fielding A, Kleinermans D, and Buss N. Pharmacokinetic interactions between sildenafil and saquinavir/ritonavir. Br J Clin Pharmacol. 2000 August; 50(2): 99-107.

[7] Schöller-Gyüre M, Debroye C, Vyncke V, et al. Effect of TMC125 on sildenafil pharmacokinetics. Abs. 45, $7^{\text {th }}$ International Workshop on Clinical Pharmacology of HIV Therapy, Lisbon, April 2006.

[8] Dingemanse J, van Giersbergen PL, Patat A, and Nilsson PN. Mutual pharmacokinetic interactions between bosentan and lopinavir/ritonavir in healthy participants. Antivir Ther, 2010, 15(2): 157-163.

[9] Humbert M, Segal ES, Kiely DG, et al. Results of European postmarketing surveillance of bosentan in pulmonary hypertension. Eur Respir J 2007; 30: 338-344.

[10] Lennox JL, DeJesus E, Lazzarin A, Pollard RB, Madruga JV, Berger DS, Zhao J, Xu X, Williams-Diaz A, Rodgers AJ, Barnard RJ, Miller MD, DiNubile MJ, Nguyen BY, Leavitt R, Sklar P; STARTMRK investigators. Safety and efficacy of raltegravirbased versus efavirenz-based combination therapy in treatmentnaive patients with HIV-1 infection: a multicentre, double-blind randomised controlled trial. Lancet. 2009 Sep 5; 374(9692): 796806.

[11] Kobayashi M Yoshinaga T, Seki T, Wakasa-Morimoto C, Brown KW, Ferris R, Foster SA, Hazen RJ, Miki S, Suyama-Kagitani A, Kawauchi-Miki S, Taishi T, Kawasuji T, Johns BA, Underwood MR, Garvey EP, Sato A, and Fujiwara T. In vitro antiretroviral properties of S/GSK 1349572, a next generation HIV integrase inhibitor. Antimicrob Agents Chemother 2011; 55: 813-21.

[12] Underwood MR Johns BA, Sato A, Martin JN, Deeks SG, and Fujiwara T. The activity of the integrase inhibitor dolutegravir against HIV-1 variants isolated from raltegravir-treated adults. J Acquir Immune Defic Syndr 2012; 61: 297-301.

[13] Castagna A Maggiolo F, Penco G, Wright D, Mills A, Grossberg R, Molina JM, Chas J, Durant J, Moreno S; the VIKING-3 Study group. Dolutegravir in Antiretroviral-Enperienced Patients With Raltegravir- and/or Elvitegravir-Resistant HIV-1: 24-Week Results of the Phase III VIKING-3 Study. JID 2014 Aug 1; 210(3): 354-62.

[14] Gulick R. HIV treatment 2020: what will it look like? Journal of the International AIDS Society 2014, 17(Suppl 3): 19528. 